\title{
Fault Diagnosis of Vehicle Engine Based on Analytic Hierarchy Process and Neural Network
}

Chuanxiang Du, Xiuling Wei

Xi'an Siyuan University; Xi'an 710038, China

Keywords: analytic hierarchy process (AHP); neural network; vehicle launching; fault diagnosis; simulation experiment

\begin{abstract}
Due to the nonlinear relationship between vehicle engine fault characteristics, traditional methods can not accurately describe the correlation characteristics between different features, it is very difficult to achieve accurate diagnosis of vehicle engine fault. In order to improve the accuracy of vehicle engine fault diagnosis, a new fault diagnosis method for vehicle engine AHP and neural network based on extracted characteristics of the vehicle engine fault diagnosis, then use AHP to determine the weight value of the vehicle engine fault diagnosis, the fault diagnosis of vehicle engine features as the input of neural network, the establishment of classification engine fault diagnosis of the vehicle, the vehicle engine fault diagnosis simulation experiment, the results show that this method improves the accuracy of engine fault diagnosis, fault diagnosis and accelerate the speed of the vehicle engine, has important practical significance.
\end{abstract}

\section{Introduction}

The vehicle engine provides power and power for the vehicle, which is the basic condition for the safe driving and operation of the vehicle. The system failure of the vehicle engine has a direct impact on the safety of the vehicle, and also affects the economy, power and reliability of the engine. With the working performance of the engine of the vehicle to improve the degree of automation, continuous improvement, its structure is becoming more and more complex, and the working environment is bad, the fault of the vehicle engine has diversity, frequency, uncertainty and destructive characteristics of the fault recognition is more and more difficult. It is of great significance to explore the reliable and accurate method of engine fault identification for the safety of human and vehicle, environmental pollution and so on[1-3].

Closely related to operating state of the vehicle engine fuel system fault and lubrication, oil, exhaust, cooling subsystem, and fuel transportation, heating, atomization, spray, combustion and many other sub processes are also closely linked, which makes the engine fault information is stochastic and uncertain n[4-6]. Aiming at the problem of vehicle engine fault diagnosis, the traditional vehicle engine troubleshooting is done by manual way. Fault diagnosis can only rely on personal experience of electrician, and the effect of vehicle engine failure is poor. With the increasing complexity of vehicle engines, the traditional fault diagnosis method is difficult to meet the requirements of vehicle engine fault diagnosis. Some experts put in a large amount of time to carry out extensive and in-depth research, and put forward a number of effective vehicle engine fault diagnosis algorithms [7]. In recent years, with the development of artificial intelligence technology, the fault diagnosis model of vehicle engine based on machine learning, fault diagnosis of the vehicle engine is modeled as a multi classification problem, so the modeling process including feature extraction and classifier construction of two steps [8]. There are many current methods of fault feature extraction, wavelet analysis in the frequency domain and time domain analysis of partial information signal, it can decompose and reconstruct the noise reduction and noise, but also can accurately detect the signal mutation, can also take the fault feature vector extraction. The main vehicle engine fault classifier based on neural network was constructed, in which the BP neural network model for distributed storage, parallel processing, adaptive, self-organizing and self-learning ability, according to the fault case study, using neural network weights to denote the weights, learning to fault diagnosis the fault 
memory, Lenovo, similar induction and pattern matching, so that we can construct the nonlinear mapping relationship between the fault and the fault symptom, has been widely used in the field of fault diagnosis $n[9-11]$.

In order to improve the accuracy of vehicle engine fault diagnosis, a new fault diagnosis method for vehicle engine AHP and neural network based on extracted characteristics of the vehicle engine fault diagnosis, then use AHP to determine the weight value of the vehicle engine fault diagnosis, the fault diagnosis of vehicle engine features as the input of neural network, the establishment of classification engine fault diagnosis of the vehicle, the vehicle engine fault diagnosis simulation experiment, the results show that this method improves the accuracy of engine fault diagnosis, fault diagnosis and accelerate the speed of the vehicle engine, has important practical significance.

\section{The Mathematical Model of Vehicle Engine Fault Diagnosis}

The fault diagnosis of vehicle engine is a certain technology to classify the working state of the vehicle engine, so as to ensure the normal work of the vehicle engine. Vehicle engine is a nonlinear system, which has many characteristics, and the interaction between features leads to complex nonlinear relationship between vehicle engine characteristics and output. It is difficult to establish a reasonable and accurate mathematical expression. The fault diagnosis of vehicle engine is $\{\mathrm{x} 1, \mathrm{x} 2, \ldots, \mathrm{xm}\}$, then the mathematical model of vehicle engine fault diagnosis is

$$
y=f\left(x_{1}, x_{2}, \cdots, x_{n}\right)
$$

In the formula, the fault diagnosis function of the vehicle engine is expressed.

Modeling of vehicle engine fault diagnosis is to find a most suitable (f), the complex nonlinear relationship between well describe the vehicle engine characteristics and output, the BP neural network has a good nonlinear learning ability, this paper uses the BP neural network for function approximation.

\section{Vehicle Engine Fault Diagnosis Feature Extraction}

\subsection{Wavelet Packet Analysis}

Wavelet packet decomposition can not only decompose the low frequency signal, and can decompose the high frequency signal, the relative $\mathrm{Yu}$ Xiaobo decomposition, wavelet packet decomposition is a fine decomposition technique, can adopt multi-level decomposition of frequency band, improve the time-frequency resolution, can be refined by extraction of vehicle engine fault characteristic frequency band. In wavelet packet decomposition, I in node (I, J) denote the number of wavelet packet decomposition. $J$ represents the sequence of the same level wavelet packet decomposition, and each node corresponds to the characteristics of vehicle engine fault signal. The two-layer wavelet packet decomposition is as shown in Figure 1.

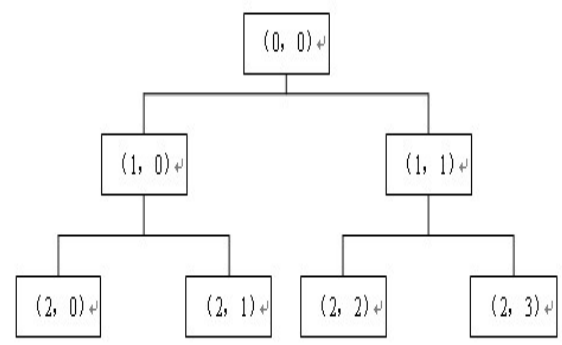

Fig.1 Schematic diagram of two layer wavelet packet decomposition

\subsection{Energy Feature Extraction of Wavelet Packets}

The fault signal of vehicle engine belongs to a non-stationary signal, and the wavelet packet decomposition algorithm is shown as follows: 


$$
\left\{\begin{array}{l}
d_{i, j, 2 m}=\frac{1}{\sqrt{2}} \sum_{l} h_{0(l-2 k)} d_{i+1, l, m} \\
d_{i, j, 2 m+1}=\frac{1}{\sqrt{2}} \sum_{l} h_{1(l-2 k)} d_{i+1, l, m}
\end{array}\right.
$$

In the formula, di,j, $m$ is the top of wavelet packet decomposition; di,j,2m and di,j, $2 m+1$ are further wavelet packet decomposition; $i$ represents the scale of wavelet packet; $j$ indicates the location of wavelet packet decomposition; $\mathrm{m}$ represents the wavelet packet frequency; 1 represents a variable, h0 and $\mathrm{h} 1$ are respectively the coefficient of multi resolution filter. The wavelet packet reconstruction algorithm is shown as follows:

$$
d_{i, j, m}=\sum_{l}\left(h_{0(l-2 k)} d_{i+1, l, 2 m}+h_{1(l-2 k)} d_{i, l, 2 m+1}\right)
$$

The wavelet packet energy spectrum refers to the result that the wavelet packet decomposition is expressed in the form of energy. The vehicle engine signal $\mathrm{x}(\mathrm{t}$ can be divided into the following wavelet packet decomposition (WT):

$$
x(t)=\sum_{j=0}^{2^{i}-1} f_{i j}\left(t_{j}\right)
$$

In the formula, fij(tj)represents the reconstruction signal of the wavelet packet of the vehicle engine signal on the $\mathrm{i}$ layer node $(\mathrm{i}, \mathrm{j})$.

Under the condition of failure, the energy of signals will vary greatly in different frequency bands, and the different fault types will have different effects on the signal energy in different frequency bands. Therefore, the vehicle engine can be diagnosed according to the energy spectrum in different frequency bands. The energy spectrum of the wavelet packet analysis can be represented as the following form:

$$
E_{i j}\left(t_{j}\right)=\int_{T}\left|f_{i j}\left(t_{j}\right)\right|^{2} d t=\sum_{j=1}^{m}\left|x_{i j}\right|^{2}
$$

In the formula, Eij(tj) represents the band energy value corresponding to the $\mathrm{j}$ node on the $\mathrm{i}$ level, and xij represents the amplitude corresponding to the discrete points of the reconstructed signal fij(tj).

Therefore, the total energy of the vehicle engine signal can be expressed as follows:

$$
E=\sum_{j=1}^{2^{i}-1} E_{i j}\left(t_{j}\right)
$$

When the vehicle engine signal is decomposed on the i layer, the percentage of the energy corresponding to the different frequency bands is shown as follows:

\subsection{Features Extraction Steps}

$$
P_{i j}=\frac{E_{i j}\left(t_{j}\right)}{E} \times 100 \%
$$

(1) The signal of vehicle engine is analyzed by wavelet packet, and each subband is obtained.

(2) The single branch reconstruction of the discrete signal of each subband is carried out to find the energy of the reconstructed signals. The signal energy corresponding to the $\mathrm{K}$ band of the $\mathrm{j}$ layer after the decomposition of the wavelet packet is Ejk, and then there is:

$$
E_{j k}=\sum_{m=0}^{L}\left|x_{k m}\right|^{2}
$$

Among them, $\mathrm{j}$ wavelet packet decomposition level; $\mathrm{k}=0,1,2, \ldots 2 \mathrm{j}-1$ decomposes the sequence number of the frequency band, $\mathrm{L}$ is the $\mathrm{K}$ band data length of the $\mathrm{j}$ layer, $\mathrm{m}$ is the discrete point of the reconstruction band of the $\mathrm{k}$ band of $\mathrm{j}$ layer, $\mathrm{xkm}$ reconstructs the amplitude of the discrete point.

(3) Normalize the energy characteristics of the signals in each band of $\mathrm{j}$ layer, and form an energy feature vector $\mathrm{t}$. 


$$
t=\left(\frac{E_{j 0}}{E}, \frac{E_{j 1}}{E}, \cdots, \frac{E_{j k}}{E}\right), \quad E=\sum_{k=0}^{2^{j}-1} E_{j k}
$$

(4) Select the most characteristic component of the data from the T, and make up the feature vector $\mathrm{T}$.

$$
T=\left(\frac{E_{j n_{0}}}{E}, \frac{E_{j n_{1}}}{E}, \cdots, \frac{E_{j n_{k}}}{E}\right)
$$

\section{Fault Diagnosis Method of Vehicle Engine Based on AHP-BPNN}

\subsection{AHP Determine the Optimal Feature}

The key step of the analytic hierarchy process is to construct the characteristic judgment matrix. In order to reduce the influence of subjective factors, to compare the characteristics of fault diagnosis of vehicle engine, constructing judgment matrix A, A matrix elements value feature representation for vehicle engine fault diagnosis results of relative importance, the vehicle engine fault diagnosis expert scoring to determine judgment matrix elements in the evaluation standards are shown in table 1.

Tab. 1 criteria for determining the assignment of elements in a matrix

$\begin{array}{cc}\text { assignment }(\mathrm{wi} / \mathrm{wj}) & \text { Explain } \\ 3 & \text { Indicating that two features are of the same importance } \\ 5 & \text { Indicates that } \mathrm{Vi} \text { features are slightly more important than } \mathrm{Vj} \\ 7 & \text { Indicates that } \mathrm{Vi} \text { features are significantly more important than } \mathrm{Vj} \\ 9 & \text { Indicates that } \mathrm{Vi} \text { features are more important than } \mathrm{Vj} \\ \text { Indicates that } \mathrm{Vi} \text { features are very important than } \mathrm{Vj}\end{array}$

According to the characteristic matrix $A W=\lambda_{\max } W$, we first get the $\mathrm{W}$ and then normalize it to get the relative importance of the corresponding feature to the upper level. Finally, we test the consistency of the judgement matrix.

The relative importance of the same level for vehicle engine fault diagnosis is calculated, and the comprehensive weight is obtained. Then the consistency of the judgement matrix is tested from high to low level. Finally, the characteristics are sorted according to the weight of the fault diagnosis characteristics of the vehicle engine.

According to the characteristics of the impact on the results of fault diagnosis of the vehicle engine weight in order to eliminate the important features, selected important features as the input of neural network, reduce the input dimensions of the neural network, simplify the network, accelerate the learning speed of neural networks, improve the vehicle engine fault diagnosis effect

\subsection{BP Neural Network}

BP neural network is a kind of error back propagation neural network. It is made up of input layer, hidden layer and output layer. It is the most widely used artificial neural network. The output of BP neural network input layer is:

$$
O_{j}^{(1)}=x(j)
$$

The input and output of the hidden layer of the neural network are:

$$
\left\{\begin{array}{l}
n e t_{i}^{(2)}(k)=\sum_{j=0}^{M} w_{i j}^{(2)} O_{j}^{(1)} \\
O_{i}^{(2)}(k)=f\left(n e t_{i}^{(2)}(k)\right)
\end{array}\right.
$$

The activation function of the neurons in the hidden layer takes positive and negative symmetric Sigmoid:

$$
f(x)=\tanh (x)=\frac{e^{x}-e^{-x}}{e^{x}+e^{-x}}
$$

The input and output of the network output layer are as follows: 


$$
\left\{\begin{array}{l}
n e t_{l}^{(3)}(k)=\sum_{i=0}^{Q} w_{l i}^{(3)} O_{i}^{(2)}(k) \\
O_{l}^{(3)}(k)=g\left(n e t_{l}^{(3)}(k)\right)
\end{array}\right.
$$

The activation function of the output layer neurons is a nonnegative Sigmoid function:

$$
g(x)=\frac{e^{x}}{e^{x}+e^{-x}}
$$

The gradient descent algorithm is used to determine the threshold and weight of the BP neural network, so that the mean square error of the vehicle engine fault diagnosis is minimized.

\subsection{Fault Diagnosis of Vehicle Engine Based on AHP-BPNN}

Step1: the fault diagnosis signal of vehicle engine is collected and the fault diagnosis feature of vehicle engine is extracted.

Step2: the analytic hierarchy process is used to calculate the comprehensive weight of vehicle engine fault diagnosis characteristics, and sort according to the importance of the characteristic weights.

Step3: according to the feature weight, screening method is used to select the characteristics of the vehicle engine fault diagnosis results.

Step4: according to the characteristics of AHP screening, we determine the number of neurons in the BPNN input layer, and take the result of vehicle engine fault diagnosis as the output of the model. The number of neurons in the hidden layer can be determined by the gradual growth method, so we can determine the topology of BPNN model.

Step5: normalization of features to eliminate the adverse effects of feature dimension differences.

Step6: initialize the BPNN parameters, and select enough vehicle engine fault diagnosis samples, use BPNN to train and learn, so as to establish the vehicle engine fault diagnosis model.

Step7: using the established model to diagnose the vehicle engine test samples, and output the result of vehicle engine fault diagnosis, and analyze its performance.

\section{Simulation Experiment}

\section{1 simulation platform}

In order to vehicle engine fault diagnosis performance analysis of AHP-BPNN, Matlab 2014 toolbox simulation test, the vehicle engine as the research object, the current vehicle engine failure varied, common are: fire engine, engine mechanical noise, air inlet system fault, overheating, test for each state of the transformer, get the signal the different states of the sample data are shown in table 1. The comparison experiment of vehicle engine fault diagnosis method of AHP and BP neural network (BPNN) is made by using the analytic hierarchy process (AHP) and the BPNN neural network.

Tab.2 sample data for vehicle engine fault diagnosis

\begin{tabular}{cccc}
\hline No. & state & training set & Validation set \\
\hline 1 & Engine fire & 30 & 20 \\
2 & Engine mechanical abnormal sound & 20 & 10 \\
3 & Intake system failure & 20 & 10 \\
4 & Fuel injection Kong Dusai & 20 & 10 \\
5 & Needle valve hindrance & 20 & 10 \\
\hline
\end{tabular}

If the data value is too large or too small, the data will lead to increased computation complexity and long training time. For that purpose, normalization and scaling to the closed interval $[0,1]$ will be done.

$$
x_{i}^{\prime}=\frac{x_{i}-x_{i \min }}{x_{i \max }-x_{i \min }}
$$

In the form, $\mathrm{t} x_{i \min }$ and $x_{i \max }$ represented the minimum and maximum value of the $\mathrm{i}$ feature, $x_{i}^{\prime}$ represents the normalized value. 
The weight of vehicle engine fault diagnosis is determined by analytic hierarchy process (AHP), and then selected according to the weight value. Then the new data set is processed according to the characteristics after filtering. The data are divided into training sample set and test sample set, and the training samples are input into the BP neural network for training.

\subsection{Results and Analysis}

The classification and diagnosis of the test samples were AHP-BPNN, AHP, BPNN, the diagnostic results obtained as shown in figure $1 \sim 3$, the average recognition rate are shown in Table 2 , from table 2 we can get the following conclusions: compared with AHP, BPNN, AHP-BPNN vehicle engine fault diagnosis result is more ideal, mainly due to the vehicle engine the working state of signal pretreatment by wavelet transform can eliminate the interference of noise on the subsequent feature extraction and classifier establishment, effectively improve the recognition of vehicle engine fault diagnosis rate, misdiagnosis rate and wrong diagnosis rate was lower, and the training time is greatly reduced, accelerate the speed of classification, comparison results show that the introduction of hierarchy the analysis method and the neural network transform can improve the vehicle engine fault diagnosis effect and reliability of fault diagnosis results of the vehicle engine.

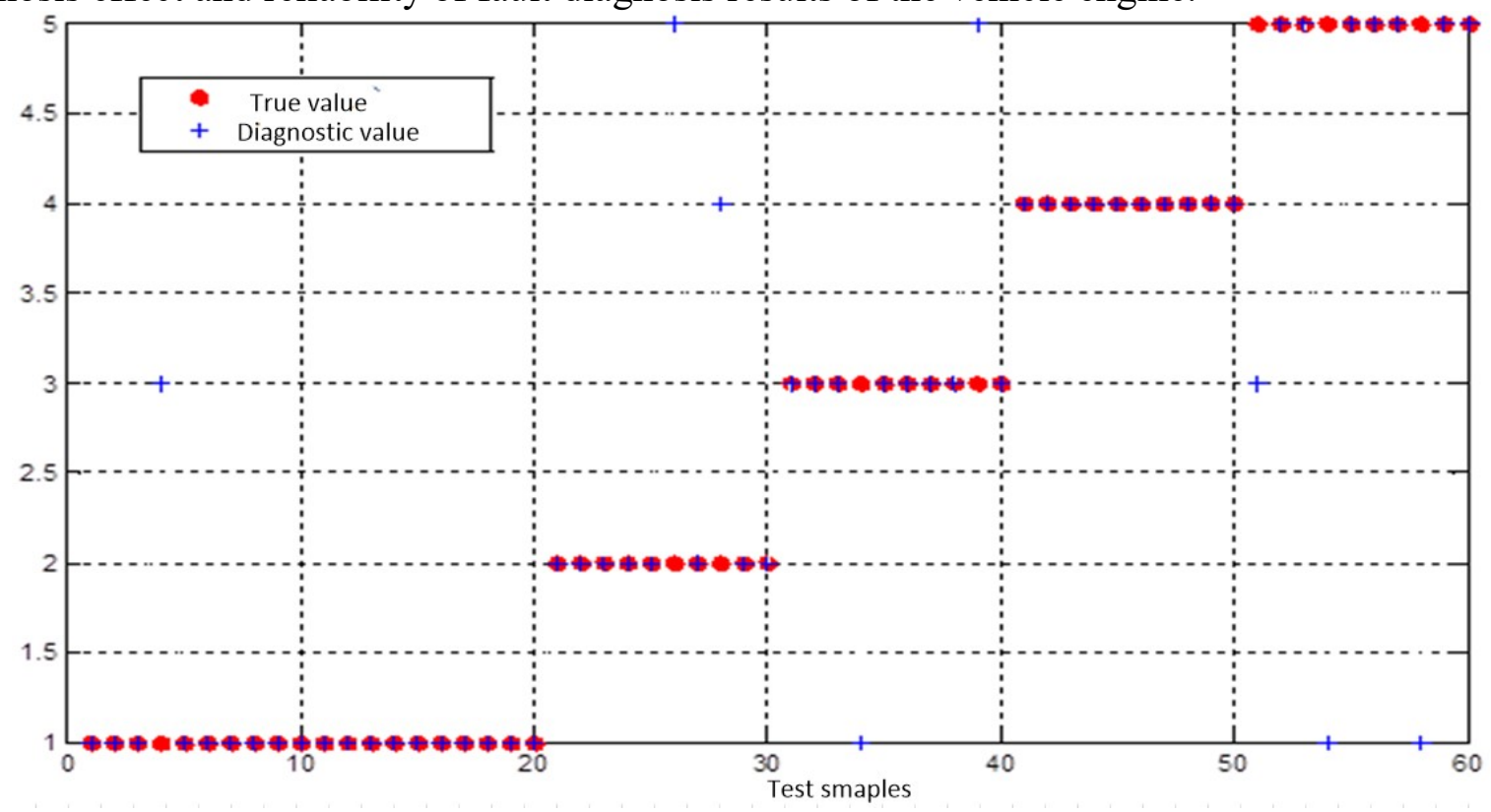

Fig.2 AHP-BPNN vehicle engine failure results

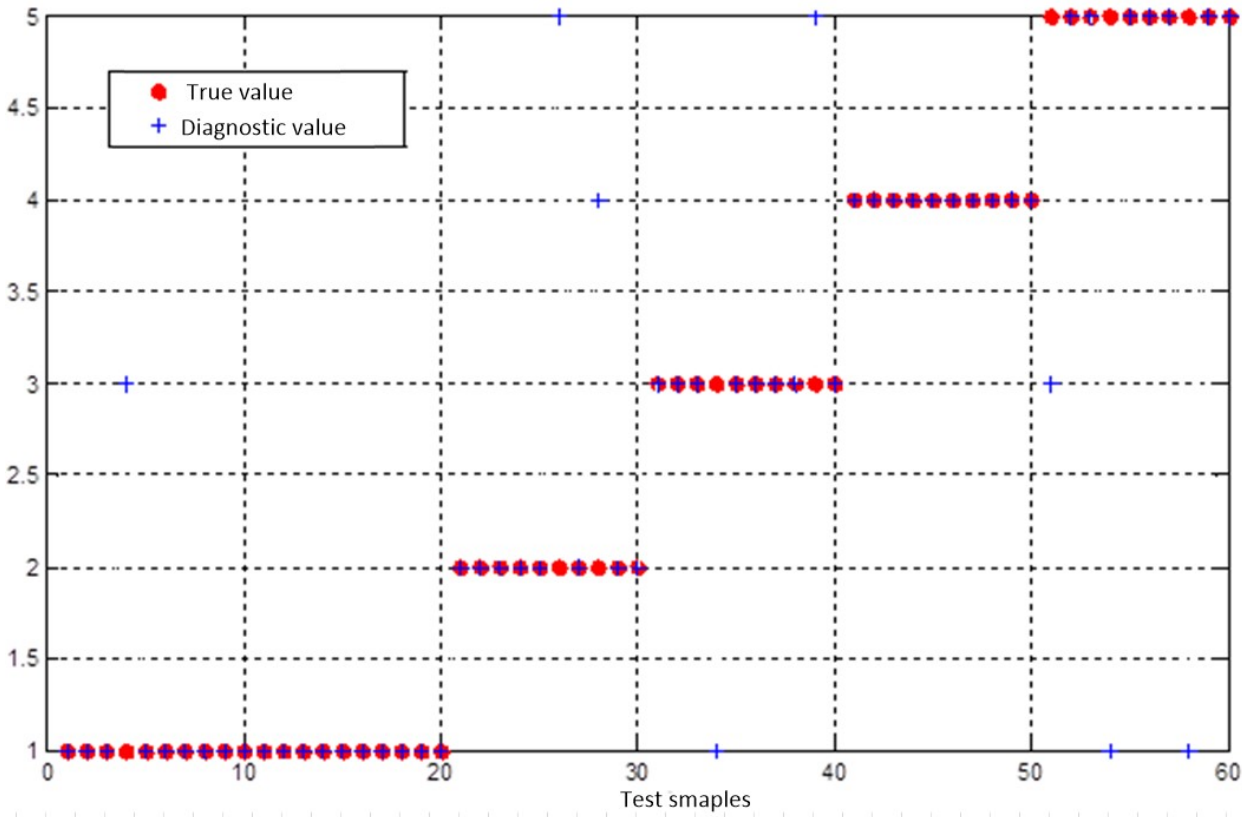

Fig.3 AHP vehicle engine failure results 


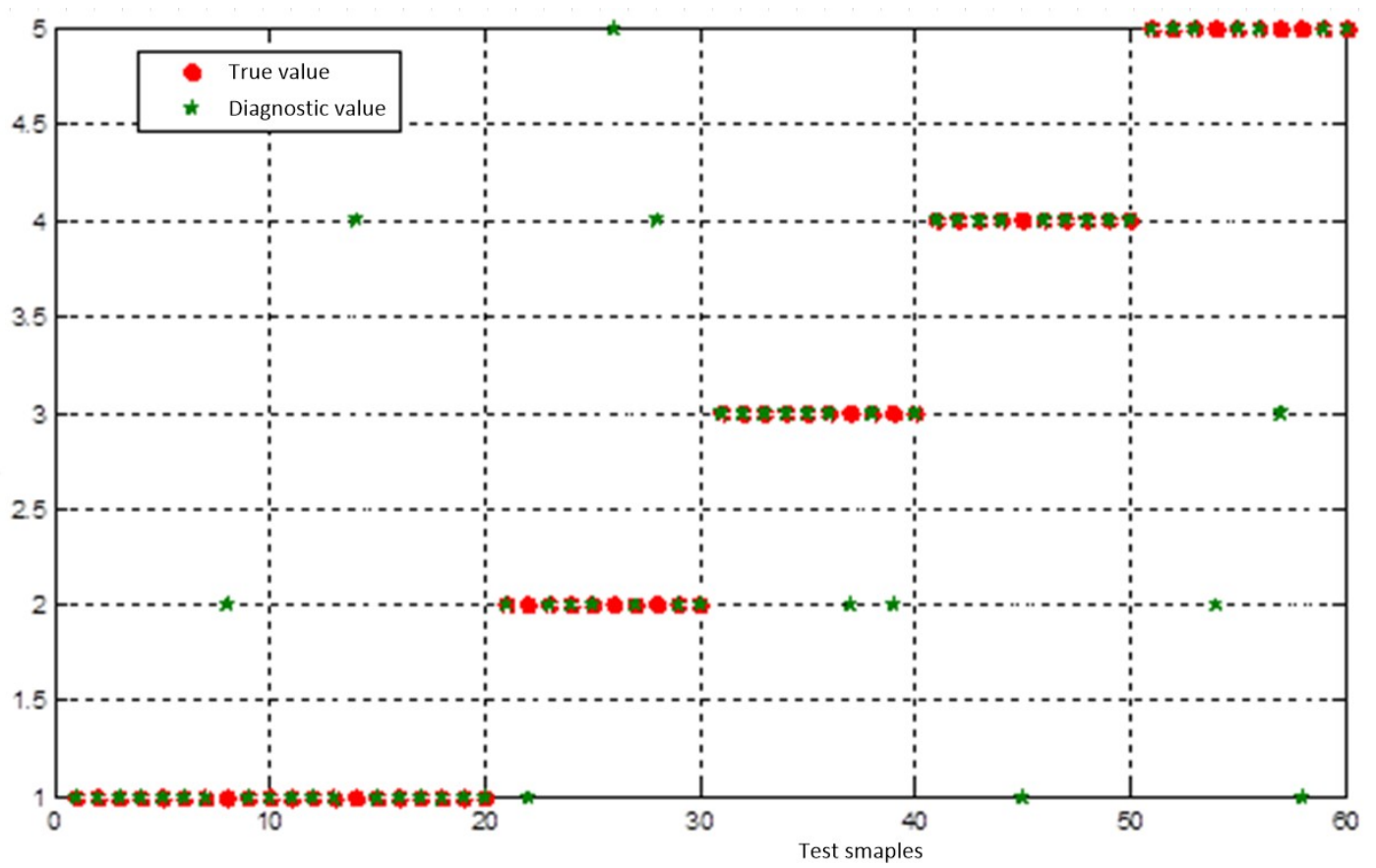

Fig. 4 BPNN vehicle engine failure results

Tab.3 Comparison of vehicle engine fault diagnosis results with different methods

\begin{tabular}{ccc}
\hline method & training time $/ \mathrm{s}$ & Diagnostic accuracy $/ \%$ \\
\hline BPNN & 19.70 & 87.14 \\
AHP & 29.44 & 85.64 \\
AHP-BPNN & 14.15 & 96.66 \\
\hline
\end{tabular}

\section{Conclusion}

In view of some problems existing in the research of vehicle engine fault diagnosis, a vehicle fault diagnosis method based on AHP-BPNN is proposed.

(1) using the wavelet packet analysis of vehicle engine fault diagnosis signal pretreatment, can eliminate the interference noise of vehicle engine fault diagnosis feature extraction is very good, is conducive to the subsequent fault diagnosis of vehicle engine, vehicle engine fault diagnosis can be obtained more satisfactory results.

(2) Using BPNN to model the mapping relationship between vehicle engine fault types and characteristics, it describes the change of vehicle engine fault diagnosis better, improves the diagnosis rate of vehicle engine fault diagnosis, and reduces the misdiagnosis rate and misdiagnosis rate.

(3) Using AHP to determine the feature weights vehicle engine fault diagnosis, solve the problem of feature weight vehicle engine fault diagnosis blindly determined, to speed up the vehicle engine fault diagnosis modeling speed, can better meet the vehicle engine fault diagnosis in real time, and broaden the scope of application.

(4) Compared with the experimental results, AHP-BPNN has the highest accuracy rate of vehicle engine fault diagnosis, indicating the effectiveness and superiority of AHP-BPNN. It has a very wide application prospect in vehicle engine fault diagnosis.

\section{Acknowledgements}

Fund project: Shaanxi Provincial Education Office Project: "Research on wireless real-time monitoring and state warning system for vehicle engines" (Number: 16JK2149) 


\section{References}

[1]. Xinhua. Analysis of automotive engine fault diagnosis technology [J]. Technology and Life, 2010, 17): 127-127.

[2]. Vong C M, Wong P K, Wong K I. Simultaneous-fault detection based on qualitative symptom descriptions for automotive engine diagnosis [J]. Applied Soft Computing, 2014, 22(0):238-248.

[3]. Hu Liping, Zhou Xuedong. Analysis of engine valve fault tree fault tree analysis [J]. Internal Combustion Engine, 2011, 1): 60-62.

[4]. Sun Yongsheng, Zhu Baoguo, Han Jun, et al. The malfunction diagnosis expert system of Cummins Engine Based on fault tree $[\mathrm{J}]$. Electromechanical Product Development and Innovation, 2011, 5: 21-23.

[5]. Zhang Wei, calamus, Hu Changhua, et al. system simulation technology for engine fault diagnosis based on confidence rule database[J]. Expert System, 2011, 7 (1): 11-15.

[6]. Liu Haibo, Li Hanwu. Research on engine fault diagnosis technology of electronically controlled automobile [J]. Journal of Harbin University of Commerce (natural science edition), 2009, 6: 318-320.

[7]. Zhang Jianjun, Zhang Li, Mu Haifang, et al. An engine fault diagnosis method based on Improved Particle Swarm Optimization BP neural network [J]. Acta agronomica Sinica, 2011, 42 (1): 198-203.

[8]. Jianmin L, Xiaolei L, Xiaoming Z, et al. Misfire Diagnosis of Diesel Engine based on Rough Set and Neural Network [J]. Procedia Engineering, 2011, 16(0): 224-229.

[9]. Vong C M, Wong P K. Engine ignition signal diagnosis with wavelet packet transform andmulti-class least squares support vector machines [J]. Expert Systems with Applications, 2011,38(7): 8563-8570.

[10]. Wang Y S, Ma Q H, Zhu Q, et al. An intelligent approach for engine fault diagnosis based on Hilbert-Huang transform and support vector machine [J]. Applied Acoustics, 2014, 75(0): 1-9.

[11]. Wu Q. Fault diagnosis model based on Gaussian support vector classifier machine [J]. Expert Systems with Applications, 2010, 37(9): 6251-6256. 